\title{
A Noisy-Channel Model for Document Compression
}

\author{
Hal Daumé III and Daniel Marcu \\ Information Sciences Institute \\ University of Southern California \\ 4676 Admiralty Way, Suite 1001 \\ Marina del Rey, CA 90292 \\ \{hdaume, marcu\}eisi.edu
}

\begin{abstract}
We present a document compression system that uses a hierarchical noisy-channel model of text production. Our compression system first automatically derives the syntactic structure of each sentence and the overall discourse structure of the text given as input. The system then uses a statistical hierarchical model of text production in order to drop non-important syntactic and discourse constituents so as to generate coherent, grammatical document compressions of arbitrary length. The system outperforms both a baseline and a sentence-based compression system that operates by simplifying sequentially all sentences in a text. Our results support the claim that discourse knowledge plays an important role in document summarization.
\end{abstract}

\section{Introduction}

Single document summarization systems proposed to date fall within one of the following three classes:

Extractive summarizers simply select and present to the user the most important sentences in a text — see (Mani and Maybury, 1999; Marcu, 2000; Mani, 2001) for comprehensive overviews of the methods and algorithms used to accomplish this.

Headline generators are noisy-channel probabilistic systems that are trained on large corpora of $\langle$ Headline, Text $\rangle$ pairs (Banko et al., 2000;
Berger and Mittal, 2000). These systems produce short sequences of words that are indicative of the content of the text given as input.

Sentence simplification systems (Chandrasekar et al., 1996; Mahesh, 1997; Carroll et al., 1998; Grefenstette, 1998; Jing, 2000; Knight and Marcu, 2000) are capable of compressing long sentences by deleting unimportant words and phrases.

Extraction-based summarizers often produce outputs that contain non-important sentence fragments. For example, the hypothetical extractive summary of Text (1), which is shown in Table 1, can be compacted further by deleting the clause "which is already almost enough to win". Headline-based summaries, such as that shown in Table 1, are usually indicative of a text's content but not informative, grammatical, or coherent. By repeatedly applying a sentence-simplification algorithm one sentence at a time, one can compress a text; yet, the outputs generated in this way are likely to be incoherent and to contain unimportant information. When summarizing text, some sentences should be dropped altogether.

Ideally, we would like to build systems that have the strengths of all these three classes of approaches. The "Document Compression" entry in Table 1 shows a grammatical, coherent summary of Text (1), which was generated by a hypothetical document compression system that preserves the most important information in a text while deleting sentences, phrases, and words that are subsidiary to the main message of the text. Obviously, generating coherent, grammatical summaries such as that produced by the hypothetical document compression system in Table 1 is not trivial because of many conflicting 


\begin{tabular}{|l|l|c|c|c|}
\hline $\begin{array}{l}\text { Type of } \\
\text { Summarizer }\end{array}$ & Hypothetical output & $\begin{array}{c}\text { Output } \\
\text { contains only } \\
\text { important info }\end{array}$ & $\begin{array}{c}\text { Output is } \\
\text { coherent }\end{array}$ & $\begin{array}{c}\text { Output is } \\
\text { grammatical }\end{array}$ \\
\hline \hline $\begin{array}{l}\text { Extractive } \\
\text { summarizer }\end{array}$ & $\begin{array}{l}\text { John Doe has already secured the vote of most } \\
\text { democrats in his constituency, which is already } \\
\text { almost enough to win. But without the support } \\
\text { of the governer, he is still on shaky ground. }\end{array}$ & & $\sqrt{ }$ \\
\hline $\begin{array}{l}\text { Headline } \\
\text { generator }\end{array}$ & mayor vote constituency governer & $\sqrt{ }$ & & \\
\hline $\begin{array}{l}\text { Sentence } \\
\text { simplifier }\end{array}$ & $\begin{array}{l}\text { The mayor is now looking for re-election. John Doe } \\
\text { has already secured the vote of most democrats } \\
\text { in his constituency. He is still on shaky ground. }\end{array}$ & & & $\sqrt{ }$ \\
\hline \hline $\begin{array}{l}\text { Document } \\
\text { compressor }\end{array}$ & $\begin{array}{l}\text { John Doe has secured the vote of most democrats. } \\
\text { But he is still on shaky ground. }\end{array}$ & $\sqrt{ }$ & $\sqrt{ }$ & $\sqrt{ }$ \\
\hline
\end{tabular}

Table 1: Hypothetical outputs generated by various types of summarizers.

goals ${ }^{1}$. The deletion of certain sentences may result in incoherence and information loss. The deletion of certain words and phrases may also lead to ungrammaticality and information loss.

The mayor is now looking for re-election. John Doe has already secured the vote of most democrats in his constituency, which is already almost enough to win.

But without the support of the governer, he is still on shaky grounds.

In this paper, we present a document compression system that uses hierarchical models of discourse and syntax in order to simultaneously manage all these conflicting goals. Our compression system first automatically derives the syntactic structure of each sentence and the overall discourse structure of the text given as input. The system then uses a statistical hierarchical model of text production in order to drop non-important syntactic and discourse units so as to generate coherent, grammatical document compressions of arbitrary length. The system outperforms both a baseline and a sentence-based compression system that operates by simplifying sequentially all sentences in a text.

\section{Document Compression}

The document compression task is conceptually simple. Given a document $D=\left\langle w_{1} w_{2} \ldots w_{n}\right\rangle$, our goal is to produce a new document $D^{\prime}$ by "dropping" words $w_{i}$ from $D$. In order to achieve this goal, we

\footnotetext{
${ }^{1} \mathrm{~A}$ number of other systems use the outputs of extractive summarizers and repair them to improve coherence (DUC, 2001; DUC, 2002). Unfortunately, none of these seems flexible enough to produce in one shot good summaries that are simultaneously coherent and grammatical.
}

extent the noisy-channel model proposed by Knight \& Marcu (2000). Their system compressed sentences by dropping syntactic constituents, but could be applied to entire documents only on a sentenceby-sentence basis. As discussed in Section 1, this is not adequate because the resulting summary may contain many compressed sentences that are irrelevant. In order to extend Knight \& Marcu's approach beyond the sentence level, we need to "glue" sentences together in a tree structure similar to that used at the sentence level. Rhetorical Structure Theory (RST) (Mann and Thompson, 1988) provides us this "glue."

The tree in Figure 1 depicts the RST structure of Text (1). In RST, discourse structures are nonbinary trees whose leaves correspond to elementary discourse units (EDUs), and whose internal nodes correspond to contiguous text spans. Each internal node in an RST tree is characterized by a rhetorical relation. For example, the first sentence in Text (1) provides BACKGROUND information for interpreting the information in sentences 2 and 3, which are in a CONTRAst relation (see Figure 1). Each relation holds between two adjacent non-overlapping text spans called NuCLEUs and SATELLITE. (There are a few exceptions to this rule: some relations, such as LIST and CONTRAST, are multinuclear.) The distinction between nuclei and satellites comes from the empirical observation that the nucleus expresses what is more essential to the writer's purpose than the satellite.

Our system is able to analyze both the discourse structure of a document and the syntactic structure of each of its sentences or EDUs. It then compresses 
the document by dropping either syntactic or discourse constituents.

\section{A Noisy-Channel Model}

For a given document $D$, we want to find the summary text $S$ that maximizes $P(S \mid D)$. Using Bayes rule, we flip this so we end up maximizing $P(D \mid S) P(S)$. Thus, we are left with modelling two probability distributions: $P(D \mid S)$, the probability of a document $D$ given a summary $S$, and $P(S)$, the probability of a summary. We assume that we are given the discourse structure of each document and the syntactic structures of each of its EDUs.

The intuitive way of thinking about this application of Bayes rule, reffered to as the noisy-channel model, is that we start with a summary $S$ and add "noise" to it, yielding a longer document $D$. The noise added in our model consists of words, phrases and discourse units.

For instance, given the document "John Doe has secured the vote of most democrats." we could add words to it (namely the word "already") to generate "John Doe has already secured the vote of most democrats." We could also choose to add an entire syntactic constituent, for instance a prepositional phrase, to generate "John Doe has secured the vote of most democrats in his constituency." These are both examples of sentence expansion as used previously by Knight \& Marcu (2000).

Our system, however, also has the ability to expand on a core message by adding discourse constituents. For instance, it could decide to add another discourse constituent to the original summary "John Doe has secured the vote of most democrats" by CONTRASTing the information in the summary with the uncertainty regarding the support of the governor, thus yielding the text: "John Doe has secured the vote of most democrats. But without the support of the governor, he is still on shaky ground."

As in any noisy-channel application, there are three parts that we have to account for if we are to build a complete document compression system: the channel model, the source model and the decoder. We describe each of these below.

The source model assigns to a string the probability $P(S)$, the probability that the summary $S$ is good English. Ideally, the source model should disfavor ungrammatical sentences and documents containing incoherently juxtaposed sentences.

The channel model assigns to any document/summary pair a probability $P(D \mid S)$. This models the extent to which $D$ is a good expansion of $S$. For instance, if $S$ is "The mayor is now looking for re-election.", $D_{1}$ is "The mayor is now looking for re-election. He has to secure the vote of the democrats." and $D_{2}$ is "The major is now looking for re-election. Sharks have sharp teeth.", we expect $P\left(D_{1} \mid S\right)$ to be higher than $P\left(D_{2} \mid S\right)$ because $D_{1}$ expands on $S$ by elaboration, while $D_{2}$ shifts to a different topic, yielding an incoherent text.

The decoder searches through all possible summaries of a document $D$ for the summary $S$ that maximizes the posterior probability $P(D \mid S) P(S)$.

Each of these parts is described below.

\subsection{Source model}

The job of the source model is to assign a score $P(S)$ to a compression independent of the original document. That is, the source model should measure how good English a summary is (independent of whether it is a good compression or not). Currently, we use a bigram measure of quality (trigram scores were also tested but failed to make a difference), combined with non-lexicalized context-free syntactic probabilities and context-free discourse probabilities, giving $P(S)=P_{\text {bigram }}(S) * P_{P C F G}(S) *$ $P_{D P C F G}(S)$. It would be better to use a lexicalized context free grammar, but that was not possible given the decoder used.

\subsection{Channel model}

The channel model is allowed to add syntactic constituents (through a stochastic operation called constituent-expand) or discourse units (through another stochastic operation called EDU-expand). Both of these operations are performed on a combined discourse/syntax tree called the DS-tree. The DS-tree for Text (1) is shown in Figure 1 for reference.

Suppose we start with the summary $S=$ "The mayor is looking for re-election." A constituent- 


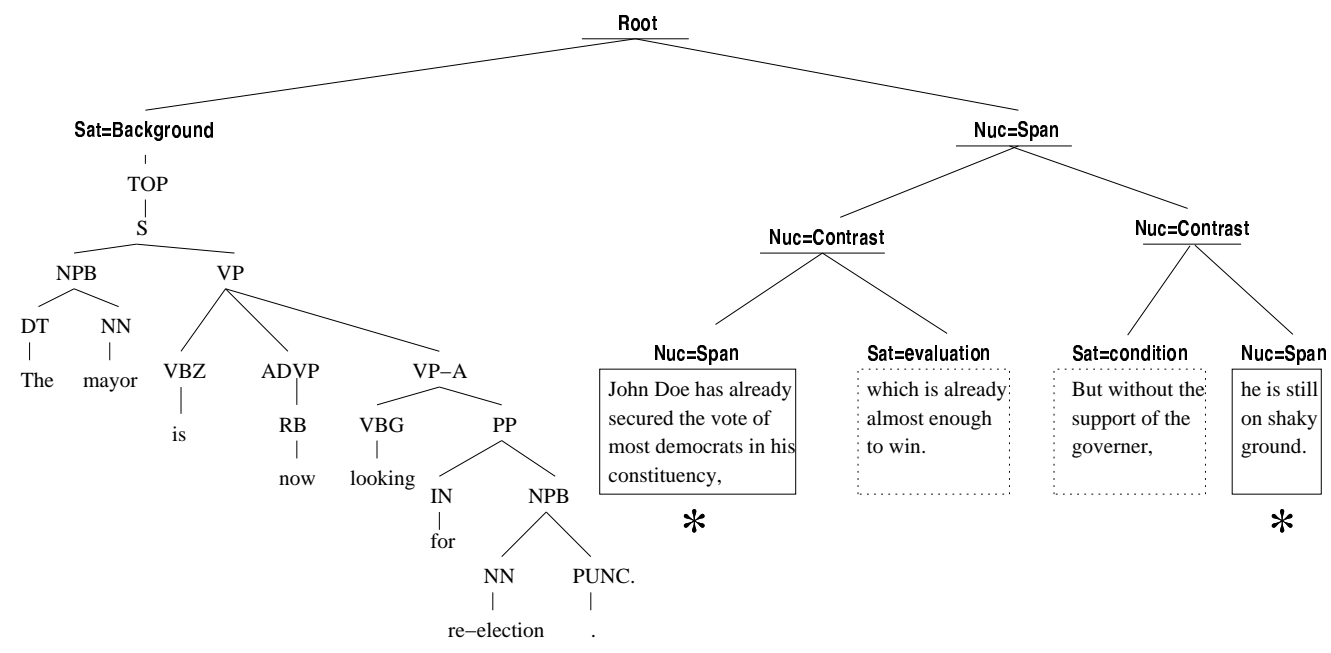

Figure 1: The discourse (full)/syntax (partial) tree for Text (1).

expand operation could insert a syntactic constituent, such as "this year" anywhere in the syntactic tree of $S$. A constituent-expand operation could also add single words: for instance the word "now" could be added between "is" and "looking," yielding $D=$ "The mayor is now looking for re-election." The probability of inserting this word is based on the syntactic structure of the node into which it's inserted.

Knight and Marcu (2000) describe in detail a noisy-channel model that explains how short sentences can be expanded into longer ones by inserting and expanding syntactic constituents (and words). Since our constituent-expand stochastic operation simply reimplements Knight and Marcu's model, we do not focus on them here. We refer the reader to (Knight and Marcu, 2000) for the details.

In addition to adding syntactic constituents, our system is also able to add discourse units. Consider the summary $S=$ "John Doe has already secured the vote of most democrats in his consituency." Through a sequence of discourse expansions, we can expand upon this summary to reach the original text. A complete discourse expansion process that would occur starting from this initial summary to generate the original document is shown in Figure 2.

In this figure, we can follow the sequence of steps required to generate our original text, beginning with our summary $S$. First, through an operation D-Project ("D" for "D"iscourse), we increase the depth of the tree, adding an intermediate
NUC $=$ SPAN node. This projection adds a factor of $P($ Nuc=Span $\rightarrow$ Nuc=Span $\mid$ Nuc=Span $)$ to the probability of this sequence of operations (as is shown under the arrow).

We are now able to perform the second operation, $D$-Expand, with which we expand on the core message contained in $S$ by adding a satellite which evaluates the information presented in $S$. This expansion adds the probability of performing the expansion (called the discourse expansion probabilities, $P_{D E}$. An example discourse expansion probability, written $P($ Nuc $=$ Span $\rightarrow$ Nuc=Span Sat=Eval $\mid$ Nuc=Span $\rightarrow$ Nuc=Span), reflects the probability of adding an evaluation satellite onto a nuclear span).

The rest of Figure 2 shows some of the remaining steps to produce the original document, each step labeled with the appropriate probability factors. Then, the probability of the entire expansion is the product of all those listed probabilities combined with the appropriate probabilities from the syntax side of things. In order to produce the final score $P(D \mid S)$ for a document/summary pair, we multiply together each of the expansion probabilities in the path leading from $S$ to $D$.

For estimating the parameters for the discourse models, we used an RST corpus of 385 Wall Street Journal articles from the Penn Treebank, which we obtained from LDC. The documents in the corpus range in size from 31 to 2124 words, with an average of 458 words per document. Each document is paired with a discourse structure that was manu- 


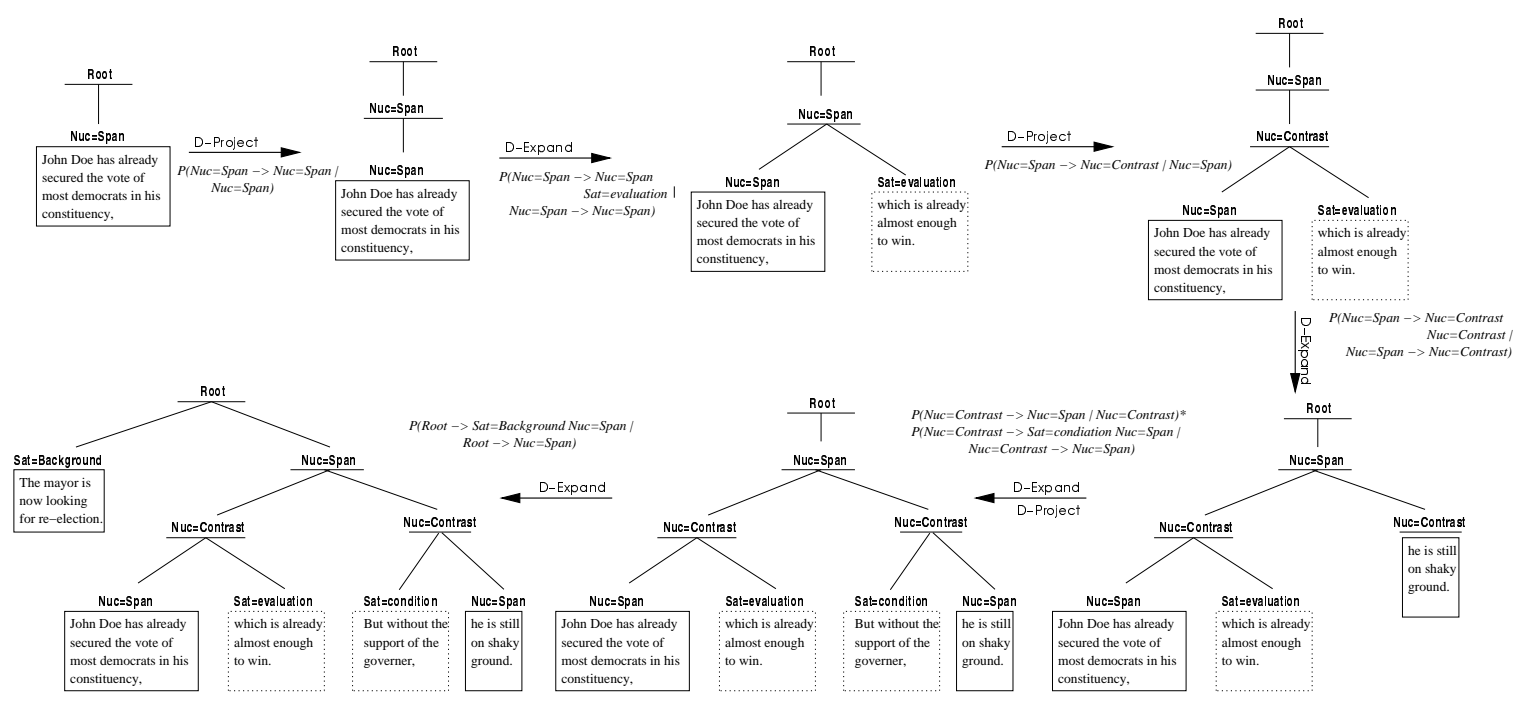

Figure 2: A sequence of discourse expansions for Text (1) (with probability factors).

ally built in the style of RST. (See (Carlson et al., 2001) for details concerning the corpus and the annotation process.) From this corpus, we were able to estimate parameters for a discourse PCFG using standard maximum likelihood methods.

Furthermore, 150 document from the same corpus are paired with extractive summaries on the EDU level. Human annotators were asked which EDUs were most important; suppose in the example DStree (Figure 1) the annotators marked the second and fifth EDUs (the starred ones). These stars are propagated up, so that any discourse unit that has a descendent considered important is also considered important. From these annotations, we could deduce that, to compress a NUC $=$ CONTRAST that has two children, NuC=SPAN and SAT=EVAluation, we can drop the evaluation satellite. Similarly, we can compress a NUC $=$ CONTRAST that has two children, SAT=CONDITION and NUC=SPAN by dropping the first discourse constituent. Finally, we can compress the ROOT deriving into SAT=BACKGROUND NUC=SPAN by dropping the SAT=BACKGROUND constituent. We keep counts of each of these examples and, once collected, we normalize them to get the discourse expansion probabilities.

\subsection{Decoder}

The goal of the decoder is to combine $P(S)$ with $P(D \mid S)$ to get $P(S \mid D)$. There are a vast number of potential compressions of a large DS-tree, but we can efficiently pack them into a shared-forest structure, as described in detail by Knight \& Marcu (2000). Each entry in the shared-forest structure has three associated probabilities, one from the source syntax PCFG, one from the source discourse PCFG and one from the expansion-template probabilities described in Section 3.2. Once we have generated a forest representing all possible compressions of the original document, we want to extract the best (or the $n$-best) trees, taking into account both the expansion probabilities of the channel model and the bigram and syntax and discourse PCFG probabilities of the source model. Thankfully, such a generic extractor has already been built (Langkilde, 2000). For our purposes, the extractor selects the trees with the best combination of LM and expansion scores after performing an exhaustive search over all possible summaries. It returns a list of such trees, one for each possible length.

\section{System}

The system developed works in a pipelined fashion as shown in Figure 3. The first step along the pipeline is to generate the discourse structure. To do this, we use the decision-based discourse parser described by Marcu (2000) ${ }^{2}$. Once we have the discourse structure, we send each EDU off to a syn-

\footnotetext{
${ }^{2}$ The discourse parser achieves an f-score of 38.2 for EDU identification, 50.0 for identifying hierarchical spans, 39.9 for nuclearity identification and 23.4 for relation tagging.
} 


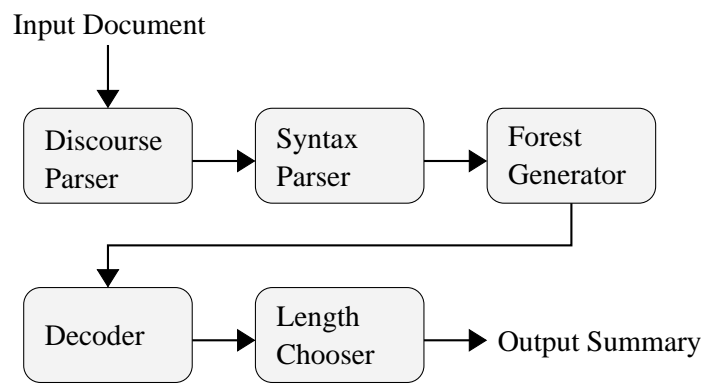

Figure 3: The pipeline of system components.

tactic parser (Collins, 1997). The syntax trees of the EDUs are then merged with the discourse tree in the forest generator to create a DS-tree similar to that shown in Figure 1. From this DS-tree we generate a forest that subsumes all possible compressions. This forest is then passed on to the forest ranking system which is used as decoder (Langkilde, 2000). The decoder gives us a list of possible compressions, for each possible length. Example compressions of Text (1) are shown in Figure 4 together with their respective log-probabilities.

In order to choose the "best" compression at any possible length, we cannot rely only on the log-probabilities, lest the system always choose the shortest possible compression. In order to compensate for this, we normalize by length. However, in practice, simply dividing the log-probability by the length of the compression is insufficient for longer documents. Experimentally, we found a reasonable metric was to, for a compression of length $n$, divide each log-probability by $n^{1.2}$. This was the job of the length chooser from Figure 3, and enabled us to choose a single compression for each document, which was used for evaluation. (In Figure 4, the compression chosen by the length selector is italicized and was the shortest one ${ }^{3}$.)

\section{Results}

For testing, we began with two sets of data. The first set is drawn from the Wall Street Journal (WSJ) portion of the Penn Treebank and consists of 16 documents, each containing between 41 and 87 words. The second set is drawn from a collection of stu-

\footnotetext{
${ }^{3}$ This tends to be the case for very short documents, as the compressions never get sufficiently long for the length normalization to have an effect.
}

dent compositions and consists of 5 documents, each containing between 64 and 91 words. We call this set the MITRE corpus (Hirschman et al., 1999). We would liked to have run evaluations on longer documents. Unfortunately, the forests generated even for relatively small documents are huge. Because there are an exponential number of summaries that can be generated for any given text ${ }^{4}$, the decoder runs out of memory for longer documents; therefore, we selected shorter subtexts from the original documents.

We used both the WSJ and Mitre data for evaluation because we wanted to see whether the performance of our system varies with text genre. The Mitre data consists mostly of short sentences (average document length from Mitre is 6 sentences), quite in constrast to the typically long sentences in the Wall Street Journal articles (average document length from WSJ is 3.25 sentences).

For purpose of comparison, the Mitre data was compressed using five systems:

Random: Drops random words (each word has a $50 \%$ chance of being dropped (baseline).

Hand: Hand compressions done by a human.

Concat: Each sentence is compressed individually; the results are concatenated together, using Knight \& Marcu's (2000) system here for comparison.

EDU: The system described in this paper.

Sent: Because syntactic parsers tend not to work well parsing just clauses, this system merges together leaves in the discourse tree which are in the same sentence, and then proceeds as described in this paper.

The Wall Street Journal data was evaluated on the above five systems as well as two additions. Since the correct discourse trees were known for these data, we thought it wise to test the systems using these human-built discourse trees, instead of the automatically derived ones. The additionall two systems were:

PD-EDU: Same as EDU except using the perfect discourse trees, available from the RST corpus (Carlson et al., 2001).

\footnotetext{
${ }^{4}$ In theory, a text of $n$ words has $2^{n}$ possible compressions.
} 


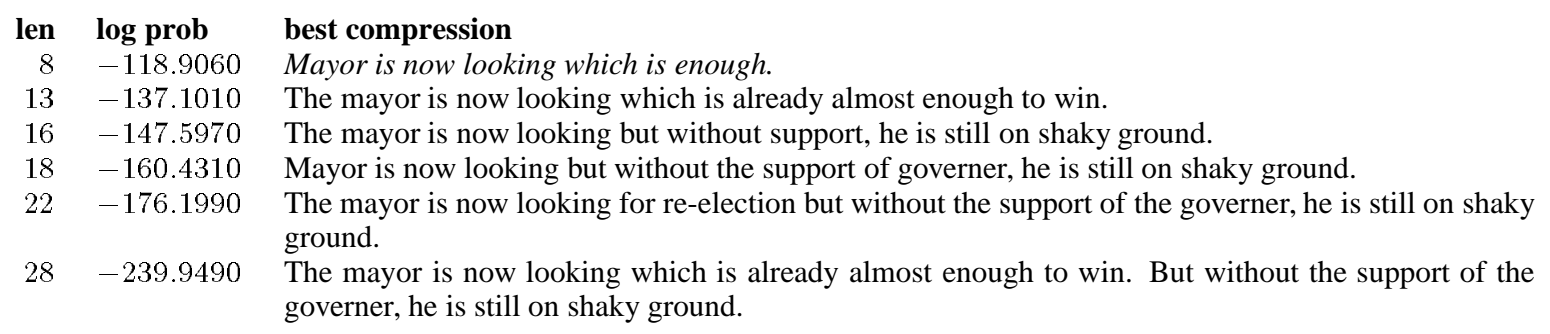

Figure 4: Possible compressions for Text (1).

PD-Sent: The same as Sent except using the perfect discourse trees.

Six human evaluators rated the systems according to three metrics. The first two, presented together to the evaluators, were grammaticality and coherence; the third, presented separately, was summary quality. Grammaticality was a judgment of how good the English of the compressions were; coherence included how well the compression flowed (for instance, anaphors lacking an antecedent would lower coherence). Summary quality, on the other hand, was a judgment of how well the compression retained the meaning of the original document. Each measure was rated on a scale from 1 (worst) to 5 (best).

We can draw several conclusions from the evaluation results shown in Table 2 along with average compression rate ( $\mathrm{Cmp}$, the length of the compressed document divided by the original length). ${ }^{5}$ First, it is clear that genre influences the results. Because the Mitre data contained mostly short sentences, the syntax and discourse parsers made fewer errors, which allowed for better compressions to be generated. For the Mitre corpus, compressions obtained starting from discourse trees built above the sentence level were better than compressions obtained starting from discourse trees built above the EDU level. For the WSJ corpus, compression obtained starting from discourse trees built above the sentence level were more grammatical, but less coherent than compressions obtained starting from discourse trees built above the EDU level. Choosing the manner in which the discourse and syntactic representations of texts are mixed should be influenced by the genre of the texts one is interested to compress.

\footnotetext{
${ }^{5}$ We did not run the system on the MITRE data with perfect discourse trees because we did not have hand-built discourse trees for this corpus.
}

\begin{tabular}{|c|c|c|}
\hline & $\begin{array}{c}\text { WSJ } \\
\text { Cmp Grm Coh Qual }\end{array}$ & $\begin{array}{c}\text { Mitre } \\
\text { Cmp Grm Coh Qual }\end{array}$ \\
\hline Random & $\begin{array}{lllll}0.51 & 1.60 & 1.58 & 2.13\end{array}$ & $\begin{array}{lllll} & 1.47 & 1.43 & 1.77 & 1.80\end{array}$ \\
\hline Concat & $0.443 .302 .98 \quad 2.70$ & $0.42 \quad 2.872 .502 .08$ \\
\hline EDU & $\begin{array}{llll}0.49 & 3.36 & 3.33 & 3.03\end{array}$ & $0.473 .403 .30 \quad 2.60$ \\
\hline Sent & 0.473 .453 .162 .88 & $\begin{array}{l}0.44 \quad 4.27 \quad 3.63 \quad 3.36\end{array}$ \\
\hline PD-EDU & 0.473 .613 .232 .95 & \\
\hline PD-Sent & $\begin{array}{lll}0.48 & 3.963 .65 & 2.84\end{array}$ & \\
\hline Hand & $\begin{array}{llll}0.59 & 4.65 & 4.48 & 4.53\end{array}$ & 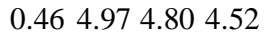 \\
\hline
\end{tabular}

Table 2: Evaluation Results

The compressions obtained starting from perfectly derived discourse trees indicate that perfect discourse structures help greatly in improving coherence and grammaticality of generated summaries. It was surprising to see that the summary quality was affected negatively by the use of perfect discourse structures (although not statistically significant). We believe this happened because the text fragments we summarized were extracted from longer documents. It is likely that had the discourse structures been built specifically for these short text snippets, they would have been different. Moreover, there was no component designed to handle cohesion; thus it is to be expected that many compressions would contain dangling references.

Overall, all our systems outperformed both the Random baseline and the Concat systems, which empirically show that discourse has an important role in document summarization. We performed $t$ tests on the results and found that on the Wall Street Journal data, the differences in score between the Concat and Sent systems for grammaticality and coherence were statistically significant at the $95 \%$ level, but the difference in score for summary quality was not. For the Mitre data, the differences in score between the Concat and Sent systems for grammaticality and summary quality were statistically significant at the $95 \%$ level, but the difference in score for 
coherence was not. The score differences for grammaticality, coherence, and summary quality between our systems and the baselines were statistically significant at the $95 \%$ level.

The results in Table 2, which can be also assessed by inspecting the compressions in Figure 4 show that, in spite of our success, we are still far away from human performance levels. An error that our system makes often is that of dropping complements that cannot be dropped, such as the phrase "for re-election", which is the complement of "is looking". We are currently experimenting with lexicalized models of syntax that would prevent our compression system from dropping required verb arguments. We also consider methods for scaling up the decoder to handling documents of more realistic length.

\section{Acknoledgements}

This work was partially supported by DARPA-ITO grant N66001-00-1-9814, NSF grant IIS-0097846, and a USC Dean Fellowship to Hal Daume III. Thanks to Kevin Knight for discussions related to the project.

\section{References}

Michele Banko, Vibhu Mittal, and Michael Witbrock. 2000. Headline generation based on statistical translation. In Proceedings of the 38th Annual Meeting of the Association for Computational Linguistics (ACL2000), pages 318-325, Hong Kong, October 1-8.

Adam Berger and Vibhu Mittal. 2000. Query-relevant summarization using FAQs. In Proceedings of the 38th Annual Meeting of the Association for Computational Linguistics (ACL-2000), pages 294-301, Hong Kong, October 1-8.

Lynn Carlson, Daniel Marcu, and Mary Ellen Okurowski. 2001. Building a discourse-tagged corpus in the framework of rhetorical structure theory. In Proceedings of the 2nd SIGDIAL Workshop on Discourse and Dialogue, Eurospeech 2001, Aalborg, Denmark, September.

John Carroll, Guidon Minnen, Yvonne Canning, Siobhan Devlin, and John Tait. 1998. Practical simplification of english newspaper text to assist aphasic readers. In Proceedings of the AAAI-98 Workshop on Integrating Artificial Intelligence and Assistive Technology.

R. Chandrasekar, Christy Doran, and Srinivas Bangalore. 1996. Motivations and methods for text simplification. In Proceedings of the Sixteenth International
Conference on Computational Linguistics (COLING '96), Copenhagen, Denmark.

Michael Collins. 1997. Three generative, lexicalized models for statistical parsing. In Proceedings of the 35th Annual Meeting of the Association for Computational Linguistics (ACL-97), pages 16-23, Madrid, Spain, July 7-12.

Proceedings of the First Document Understanding Conference (DUC-2001), New Orleans, LA, September.

Proceedings of the Second Document Understanding Conference (DUC-2002), Philadelphia, PA, July.

Gregory Grefenstette. 1998. Producing intelligent telegraphic text reduction to provide an audio scanning service for the blind. In Working Notes of the AAAI Spring Symposium on Intelligent Text Summarization, pages 111-118, Stanford University, CA, March 2325.

L. Hirschman, M. Light, E. Breck, and J. Burger. 1999. Deep read: A reading comprehension system. In Proceedings of the 37th Annual Meeting of the Association for Computational Linguistics.

H. Jing. 2000. Sentence reduction for automatic text summarization. In Proceedings of the First Annual Meeting of the North American Chapter of the Association for Computational Linguistics NAACL-2000, pages 310-315, Seattle, WA.

Kevin Knight and Daniel Marcu. 2000. Statistics-based summarization - step one: Sentence compression. In The 17th National Conference on Artificial Intelligence (AAAI-2000), pages 703-710, Austin, TX, July 30th - August 3rd.

Irene Langkilde. 2000. Forest-based statistical sentence generation. In Proceedings of the 1st Annual Meeting of the North American Chapter of the Association for Computational Linguistics, Seattle, Washington, April 30-May 3.

Kavi Mahesh. 1997. Hypertext summary extraction for fast document browsing. In Proceedings of the AAAI Spring Symposium on Natural Language Processing for the World Wide Web, pages 95-103.

Inderjeet Mani and Mark Maybury, editors. 1999. Advances in Automatic Text Summarization. The MIT Press.

Inderjeet Mani. 2001. Automatic summarization.

William C. Mann and Sandra A. Thompson. 1988. Rhetorical structure theory: Toward a functional theory of text organization. Text, 8(3):243-281.

Daniel Marcu. 2000. The Theory and Practice of Discourse Parsing and Summarization. The MIT Press, Cambridge, Massachusetts. 